\title{
MAPPING OF SODIUM VOID EFFECT AND DOPPLER CONSTANT IN ESFR-SMART CORE WITH MONTE CARLO CODE SERPENT AND DETERMINISTIC CODE ERANOS
}

\author{
Jiri Krepel ${ }^{1}$ and Valeria Raffuzzi ${ }^{1}$ \\ ${ }^{1}$ Paul Scherrer Institute \\ Forschungsstrasse 111, 5232 Villigen, Switzerland \\ jiri.krepel@psi.ch, valeria.raffuzzi@psi.ch
}

\begin{abstract}
The Sodium Fast Reactor is one of the most technologically developed Gen-IV reactors, which can close the nuclear fuel cycle. Its criticality safety directly depends on the sodium void effect and Doppler constant. Hence the knowledge of their local distribution is important. These coefficients can be mapped by deterministic or Monte Carlo codes, where the latter provide higher modeling accuracy, but are also strongly computer demanding and subject to stochastic noise issues. In this study, the void effect and Doppler constant have been enumerated for the ESFR core by Serpent 2 and ERANOS 2 codes, preserving a six-batch operation scheme. The Serpent code was coupled to the Python script BBP to simulate batch-wise operation in a radially infinite inner core configuration; the ERANOS code was applied to the whole core geometry and the batch-wise operation was simulated by the EQL3D routine. Sodium void effect and Doppler constant spatial maps with different levels of refinement were produced, as well as the time evolution of the integral coefficients during the transition from initial cycle to equilibrium cycle. Both codes indicate deterioration of these coefficients during the transition. The equilibrium cycle performance of the inner core zone from the ERANOS calculation was compared with Serpent results and they showed reasonable agreement. For very fine mapping, the Monte Carlo method employed was computationally very demanding and the enumerated effect was lower than the stochastic noise. In general, the Serpent model practically excludes modeling assumptions and produces reliable results for reasonably sized maps, which can be combined if needed with the high spatial resolution results obtained by ERANOS simulations.
\end{abstract}

KEYWORDS: ESFR-SMART, Equilibrium cycle

\section{INTRODUCTION}

Detailed knowledge of temperature and density feedback coefficients and their local distribution is crucial for the safety of any nuclear reactor. This statement is especially relevant for a Sodium cooled Fast Reactor (SFR), where the sodium density effect is usually positive and an Unprotected Loss Of Flow (ULOF) transient may result in a reactivity excursion. The coolant density effect is positive in all iso-breeding fast reactors except the molten salt reactor [1], where coolant and fuel are mixed. The sodium coolant density effect is stronger than for gas coolants, but weaker than for lead or lead-bismuth coolants. Nonetheless, compared to these heavy liquid metals, the margin between operating temperature and sodium boiling point is much smaller $(\approx 290 \mathrm{~K})$. The positive reactivity introduced by sodium boiling is the major safety risk in the case of an ULOF transient. From a neutronics perspective, the reduced sodium density decreases the neutrons' scattering in the core and the resulting spectrum hardening causes a reactivity increase. At the 
same time, reduced scattering increases the neutron leakage at the core periphery. The strength of the sodium void effect depends on the core design. It is lower in cores with higher leakage. In recent designs [2][3][4] the positive void effect is reduced by minimizing the sodium share in the core lattice and by maximizing the importance of neutron leakage. This is typically achieved by introducing an upper sodium plenum followed by a neutron absorber [2][5]. The plenum effect is amplified by increasing the neutron importance in the upper part of the core with one [4] or two [3] radial blankets in the bottom half of the core. As a result, the local void map is crucial for proper simulation and understanding of the ULOF transient behavior. It was already shown that the local void effect ranges from strongly positive (core center) to strongly negative (sodium plenum) values and that the overall effect is not a linear combination of these components [6]. Furthermore, the Doppler effect is interacting with the void effect and the spectral shift caused by one effect influences the other.

This study was carried out as a part of the H2020 ESFR-SMART project and the ESFR-SMART core was used as a reference [4]. A six-batch reloading scheme was adopted and the equilibrium End Of Cycle (EOC) state [12] was used as a reference.

Two calculation routes have been applied to enumerate the sodium void worth map and Doppler constant map. These are the ERANOS 2 code coupled with the EQL3D procedure and the Serpent 2 code coupled with the Batch Burnup Procedure (BBP). The BBP model was based on a radially infinite inner core layout. The burnup distribution was adopted from a previous study [7] and recalculated with higher precision, including the safety coefficients evolution. The EQL3D procedure was applied to the full core geometry. As such, these results are not directly comparable and a certain normalization is needed for inner core zone evaluation. This zone corresponds to the cooling group 1 [12] defined by the project.

\section{SIMULATED CORE AND METHODOLOGY}

\subsection{ESFR-SMART core}

The ESFR-SMART core layout is presented in [8]. The six-batch reloading scheme was selected based on the design fuel burnup and annual core reloading and revision. From purely neutronic perspectives, fewer batches would be also possible. The reloading map was proposed with the purpose of minimizing local average burnup oscillations for each three neighboring assemblies. Since there are 6 batches in the core, each 3 neighboring assemblies can theoretically form 21 different triplet combinations from 1-1-1 to 1-66. The burnup of these triplets evolves during the 6-years presence in the core (left Fig. 1).
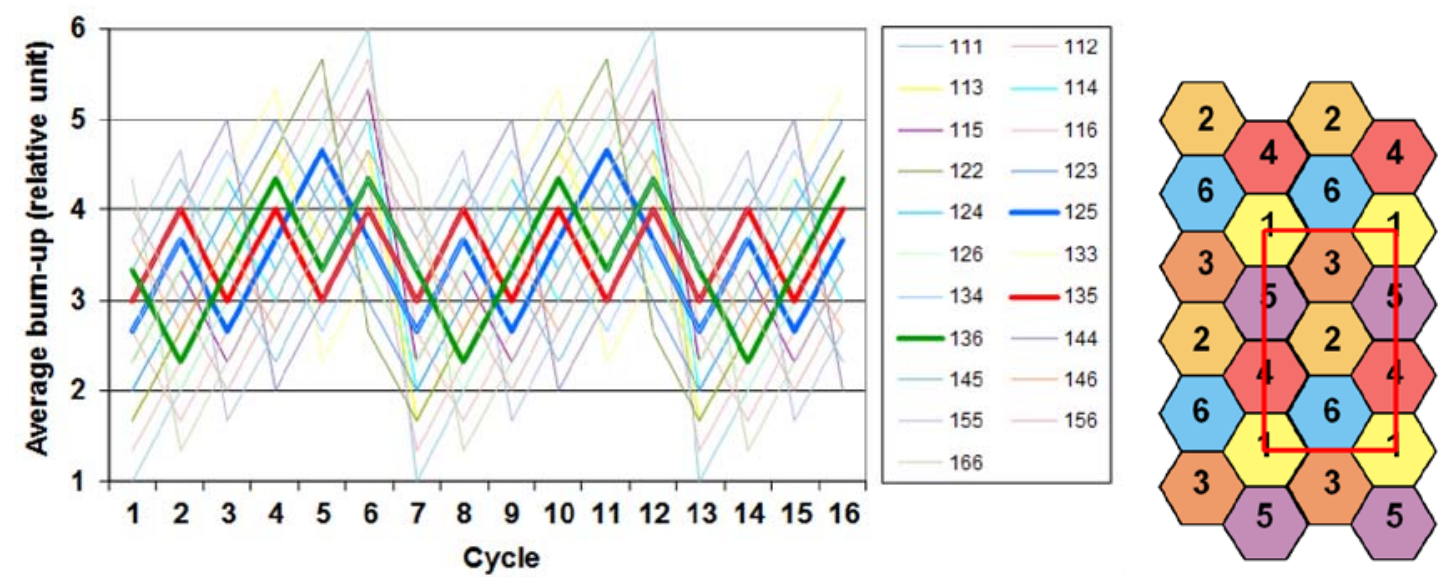

Figure 1. Average burn-up of three neighboring assemblies for all 21 possible combinations (left) and the radial layout of assembly reloading schemes for ESFR-SMART (right). 
For instance, 1-1-1, 2-2-2, 3-3-3, 4-4-4, 5-5-5, and 6-6-6 represent only the evolution of one triplet combination. Assuming that the reactivity swing of each assembly is a linear function of burnup and that reactivity is additive, these burnup oscillations represent well the reactivity oscillations. The least oscillatory triplets in the six-batch scheme are 1-2-5, 1-3-5, and 1-3-6.

The minimal repetitive pattern of assemblies in the reloading scheme is indicated by the red frame on the right side of Fig. 1. The Serpent 2 radially infinite model was based on such minimal lattice, and periodic boundary conditions were applied. The average burnup is constant and equal to 3.5 relative units. Nonetheless, there are six different triplets oscillating between 3-4, 2.3-4.3, or 2.7-4.7. The most oscillatory are the triplets $2-3-5$ and 2-4-5. Hence, the resulting reactivity oscillations obtained by later simulations will be uneven between batches.

\subsection{Evaluated safety coefficients}

In this work, the Doppler constant and void effect were calculated by direct calculations, i.e. comparing the nominal state with a perturbed state. By definition, safety feedbacks are computed as described by Eq. 1 .

$$
K_{D}=\frac{\rho_{\text {pert }}+\rho_{\text {nom }}}{\ln \left(T_{\text {pert }} / T_{\text {nom }}\right)}, \Delta \rho_{v}=\rho(\text { voided zone })-\rho(\text { flooded zone })
$$

where $K_{D}$ is the Doppler constant, $\rho$ is the core reactivity, $T$ is fuel temperature in Kelvin and $\Delta \rho_{\mathrm{v}}$ is the void effect. The uncertainties of the safety coefficients from Eq. 1, necessary to evaluate Serpent results, were calculated as in Eq. 2, derived applying the law of propagation of errors:

$$
\sigma_{D}=\frac{\sqrt{\frac{e r r_{\text {nom }}^{2}}{k_{\text {nom }}^{4}}+\frac{e r r_{\text {pert }}^{2}}{k_{\text {pert }}^{e}}}}{\ln \left(T_{\text {pert }} / T_{\text {nom }}\right)}, \sigma_{\text {void }}=\sqrt{\frac{\text { err }_{\text {nom }}^{2}}{k_{\text {nom }}^{4}}+\frac{e r r_{\text {pert }}^{2}}{k_{\text {pert }}^{4}}}
$$

where $k$ is the effective multiplication factor and err is the error of the Serpent stochastic calculation. These coefficients were evaluated for different zones at the EOC state. Additionally, their integral values were calculated during the evolution from beginning of life to the EOC state at equilibrium. The capabilities of the BBP script to manipulate Serpent inputs were extended for such calculations, while the EQL3D ERANOS procedure has this feature already integrated.

\subsection{Serpent and BBP script}

Serpent is a Monte Carlo reactor physics code developed by J. Leppanen for VTT Technical Research Centre of Finland and released in 2008 [9]. It is suitable for high precision transport and burnup calculations due to the continuous-energy cross-sections structure. However, good statistics come at the cost of a very high number of neutrons simulated, hence elevated CPU time. This is particularly true in the case of large systems with complex geometries. Very good statistics are also required for the study of local properties of large systems: as a consequence, Serpent is unsuitable for these types of calculations in favor of deterministic codes. In this work, ENDF/B-VII.0 nuclear data libraries were used for Serpent 2 simulations.

To simulate the transition from initial to equilibrium state, an external routine named BBP was coupled to Serpent. Originally, the routine was coded in Ruby and applied to the pebble-bed reactor HTR-PM [10]. Subsequently, it has been translated to Python and adapted for application to solid [7] and liquid fuel reactors. The goal of BBP is to automatize batch-wise refueling and, if needed, fuel reshuffling. The algorithm of the routine is schematized in Fig. 2. At the beginning of each cycle, the routine manipulates the input files for the Serpent simulation. 
The main input file is completed by overwriting input parameters like number of neutrons, active and inactive cycles and burnup time steps. The fuel material input file, is also prepared: the fuel composition is copied from the output of the previous cycle simulation. Then the most burnt fuel is identified and replaced with fresh fuel. In the next step, the routine calls Serpent to run the burnup calculation. The whole process is repeated for as many cycles as requested by the user. As a final step, the outputs from every cycle are processed and arranged in compact output files.

\subsection{ERANOS2 and EQL3D procedure}

The EQL3D procedure [11] simulates the reactor batch burnup by assembly shuffling or refueling at a given position. It was developed to simulate the equilibrium state of an open or closed fuel cycle. In both cases, the reactor operation is explicitly simulated under specified periodic fuel management until the open or closed cycle equilibrium is reached. EQL3D is based on the ERANOS2 code and was used with JEFF3.1 data library.

The mapping of void effect and Doppler constant was done for different levels of refinement. The most detailed maps require simulations where one zone perturbation causes a reactivity change of a few PCM

(Per Cent Mille). The methodology for simulating such small changes by direct calculation is described in [6]. The same restart file is used for nominal and perturbed cases and the calculation is stopped after a fixed number, e.g. 25, of iterations. These two calculations as such are not fully converged. The difference between them has, however, already converged and seems meaningful. Full mathematical proof of this method was not carried on. Some explanation and background is shown in [6].

\section{TRANSITION TO THE EOC STATE}

In order to assess the capability of the reduced Serpent model to simulate the inner core features, selected properties as reactivity, global void effect (active fissile core plus upper plenum) and Doppler constant are compared in Fig. 3. The absence of radial leakage in the radially infinite Serpent model has a strong influence on all three properties. The trends are similar for both models in all three cases. The reactivity is shifted up in the radially infinite case. The void effect is more negative in the radially infinite lattice, while the opposite happens to the Doppler constant. Surprisingly, both the void effect and Doppler constant of the whole core calculated at the EOC state are higher by the same factor of 1.54 in ERANOS. This number was thus used for Serpent results renormalization in the safety coefficient maps. As already mentioned, the BBP radially infinite model is most representative for the inner core, i.e. the cooling group 1 in the ESFR project. For results in other cooling groups please refer to the reference [12].

In the Serpent model, the set-up used was 50000 neutrons per cycle, 500 active and 50 inactive cycles. 

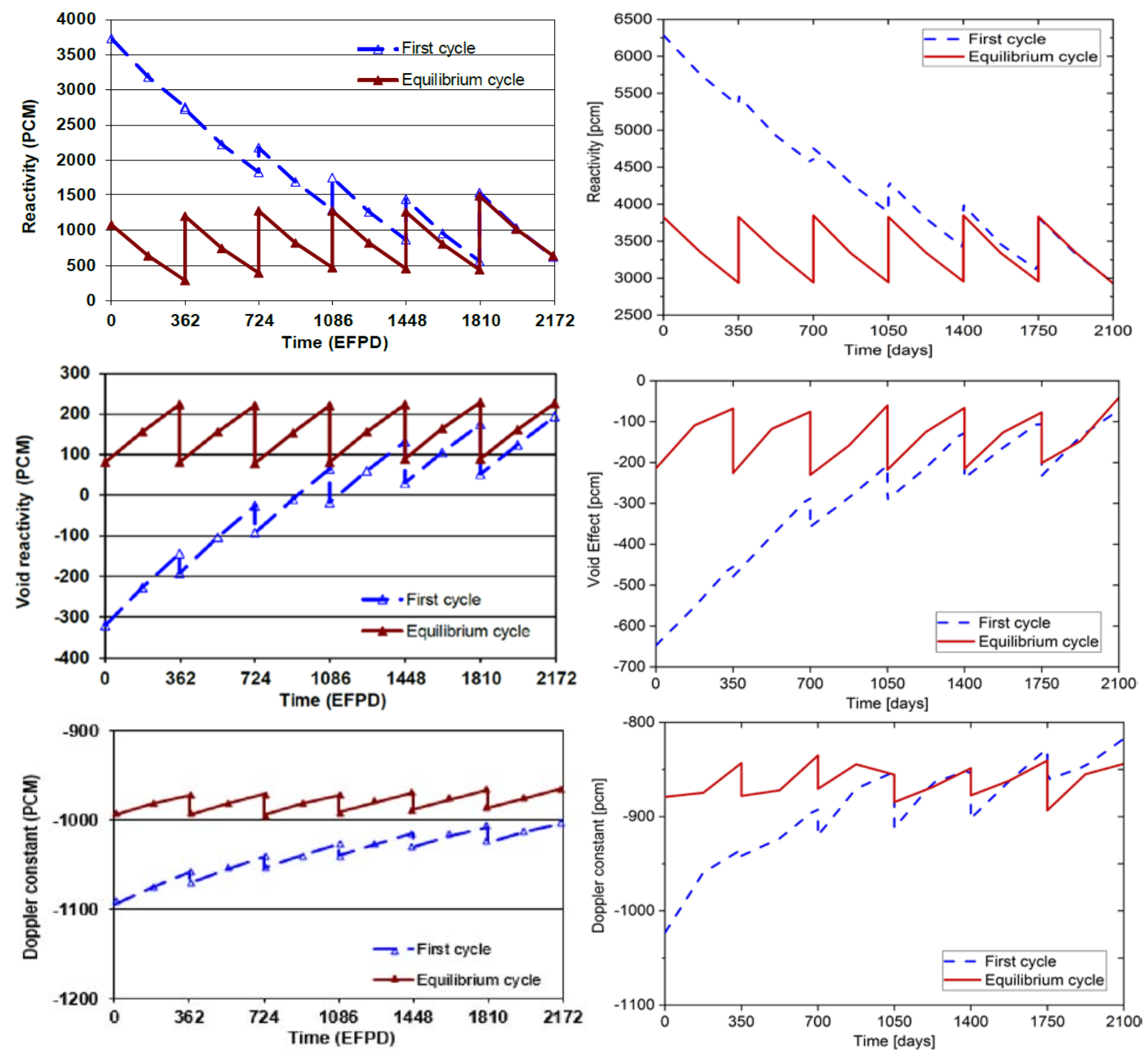

Figure 3. Reactivity (top), void effect (middle) and Doppler constant (bottom) evolution simulated with ERANOS (left) and Serpent (right).

\section{MAPPING OF THE COEFFICIENTS}

As a first option, all six fuel batches in the inner core have been perturbed simultaneously. For Doppler constant calculations the core was divided into 1, 2, 4, 10, and 20 nodes. For void reactivity the sodium plenum was also accounted for, and the inner fuel was divided into 1, 3, 8, 17, and 27 nodes. In the Serpent model, the set-up used was 20000 neutrons per cycle, 300 active and 50 inactive cycles in both of the mapping cases. The results are shown in Fig. 4 as color maps and in Fig. 5 as axial profiles. To be able to compare the results from the two codes, the radially infinite Serpent model results were divided by 1.54 for both coefficients. The color scheme in these maps was locked to the physical range. Outliers were highlighted in red or dark blue. For a coarse discretization, Serpent results are comparable to ERANOS results. However, in the 20 nodes case for the Doppler constant the reactivity change is comparable with the stochastic error, thus strong stochastic noise is generated. This behavior can be observed in Fig. 5. Since the local void effect is stronger, fewer stochastic oscillations are observed. 

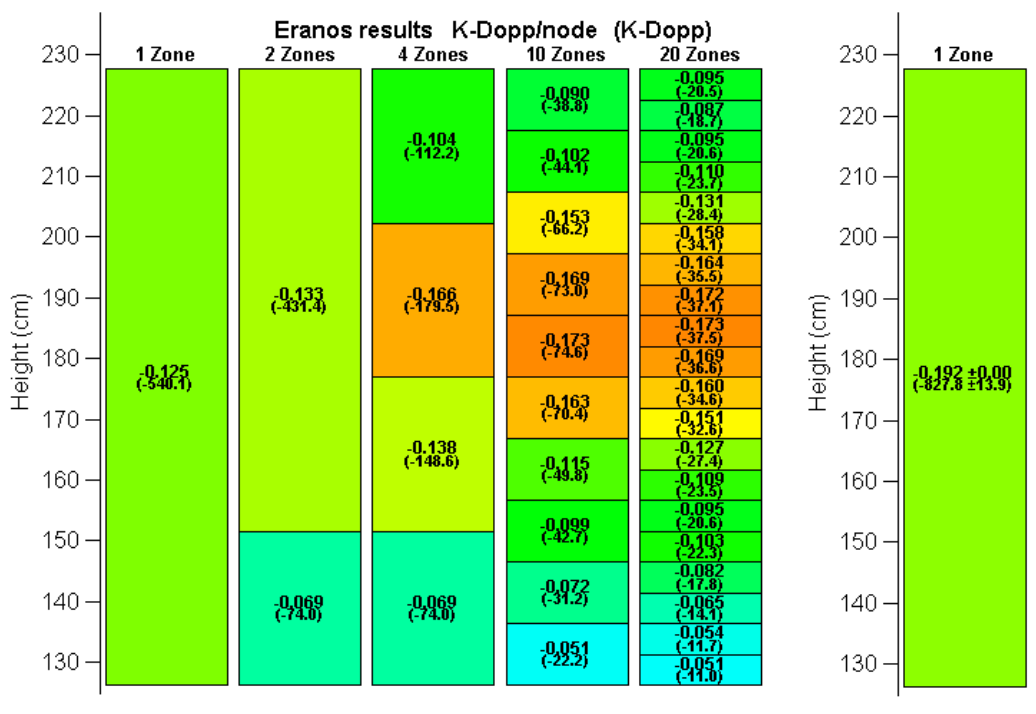

Serpent results K-Doppinode (K-Dopp)
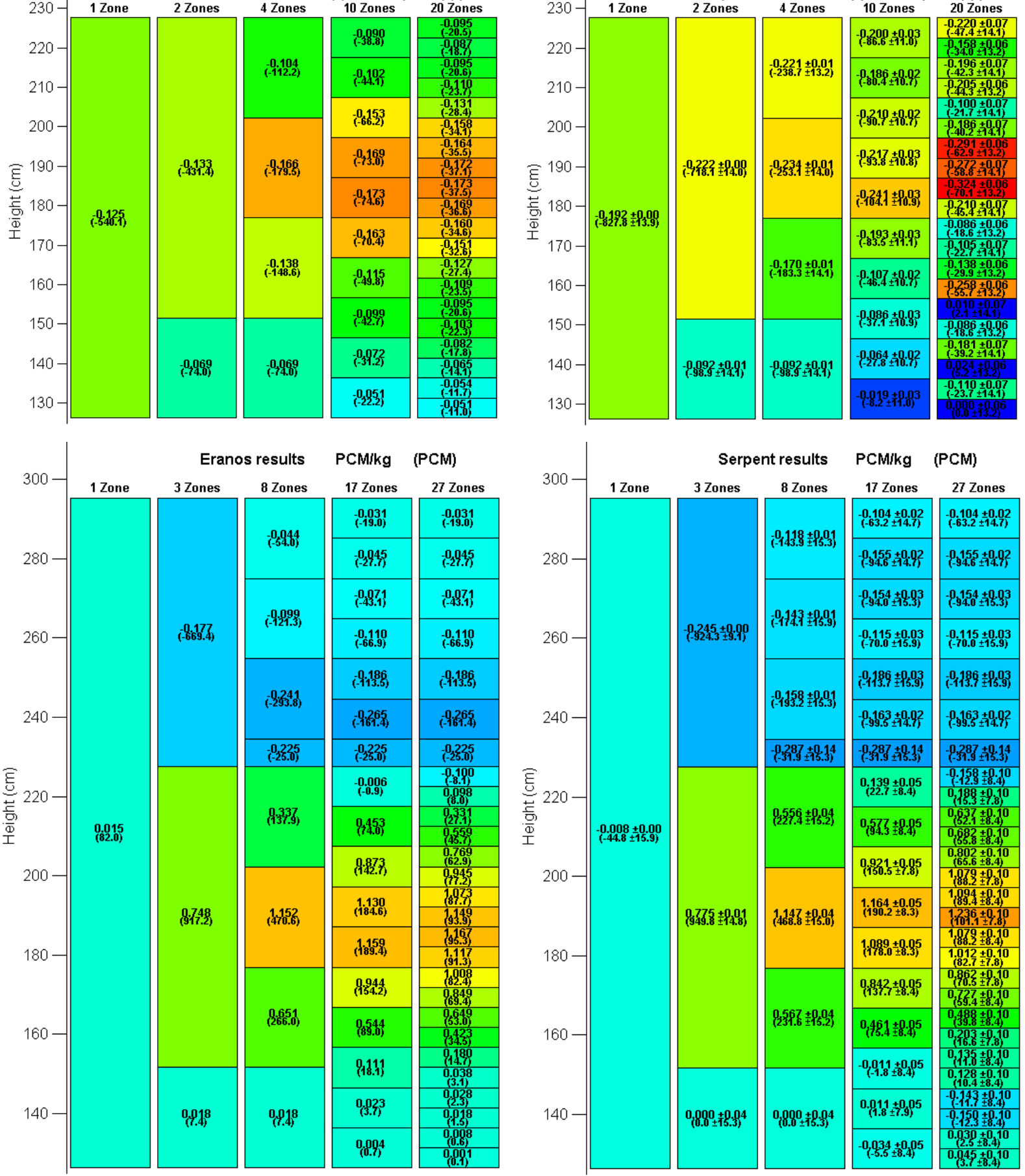

Figure 4. Doppler constant (top) and void reactivity (bottom) mapping using the full core ERANOS model (left) and the radially infinite Serpent model (right).

\section{BATCH-WISE MAPPING OF THE COEFFICIENTS}

A similar nodalization as in the previous section was used for batch-wise mapping, where each group of assemblies was perturbed separately. The mapping produced by the Serpent model for the Doppler constant faces strong stochastic behavior, because the selected accuracy is lower than the effect itself (Fig. 6 and 7). 

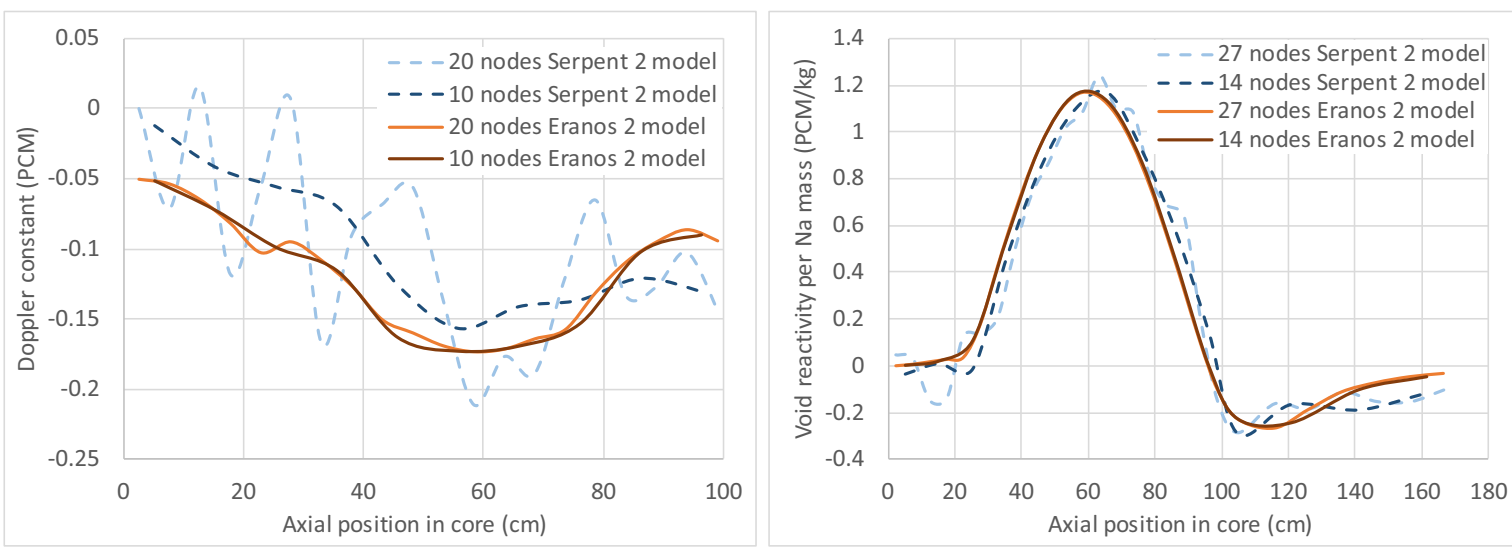

Figure 5. Doppler constant (left) and void reactivity (right) distribution along the axial height.

The void reactivity per batch can be simulated by Serpent code for 8 axial nodes in each assembly, providing still reasonable values. ERANOS can simulate both the Doppler constant and void reactivity distribution with the same accuracy for respectively 20 and 28 axial nodes in each fuel batch.
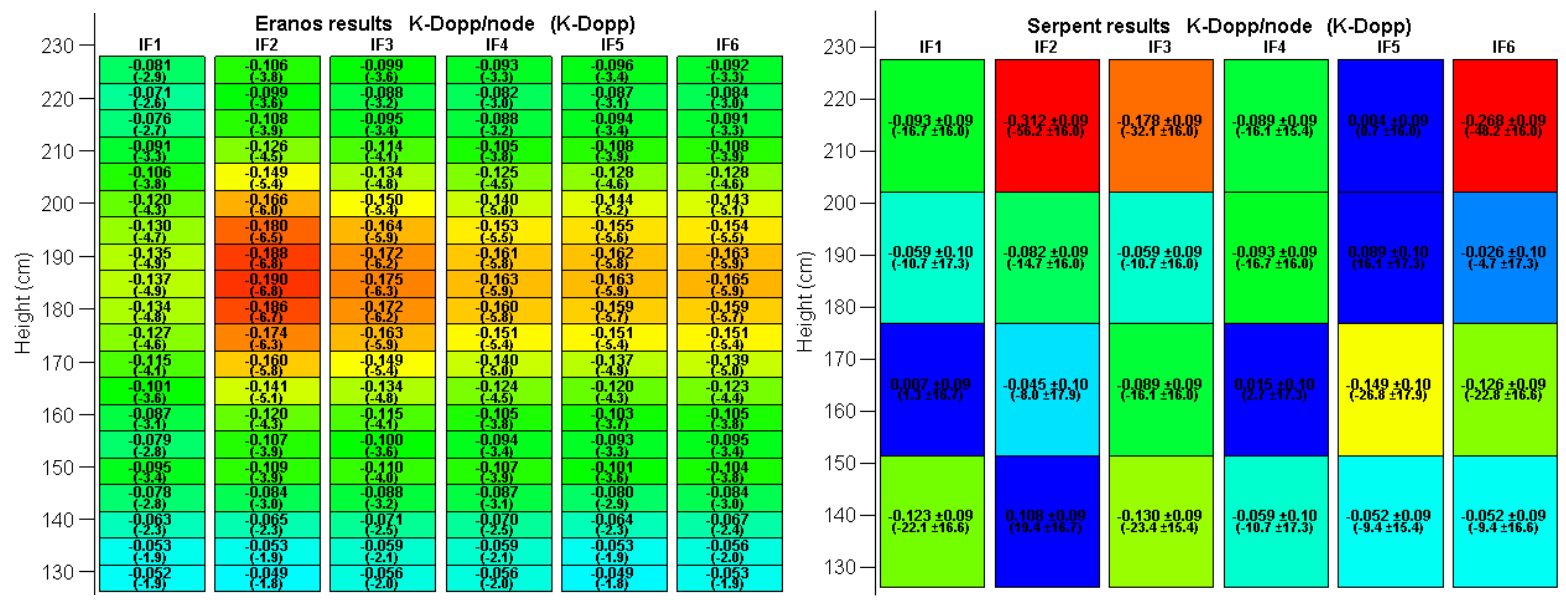

Figure 6. Doppler constant mapping for the six-batch core layout using the full core ERANOS model (left) and the radially infinite Serpent model (right).

\section{SUMMARY AND CONCLUSIONS}

In this work, two methodologies were applied to evaluate ESFR inner core performances at the equilibrium state. Those included the application of the deterministic code ERANOS2 and of the stochastic code Serpent2. Monte Carlo codes can generally produce high precision results, at the cost of high computational times. To reduce the computational resources utilization, the Serpent model was based on a fundamental six-assembly lattice, with periodic boundary conditions applied. On the other hand, the ERANOS model included the full ESFR core. The evolution of the core reactivity, void effect and Doppler constant from initial to equilibrium state showed very good agreement between the two codes. The absolute values of the feedbacks in the core region differed by a factor of 1.54 both for the void effect and the Doppler constant. Local mapping of the safety feedbacks with different levels of refinement was performed as well. While ERANOS can simulate fine maps with high accuracy, Serpent is not appropriate to compute reactivity coefficients that are close to zero, i.e. the Doppler constant of small fuel zones, since one standard deviation of statistical uncertainty may make the coefficient positive or negative. Therefore, the Serpent model is not suitable to reproduce ESFR inner core features when a fine nodalization is adopted. 

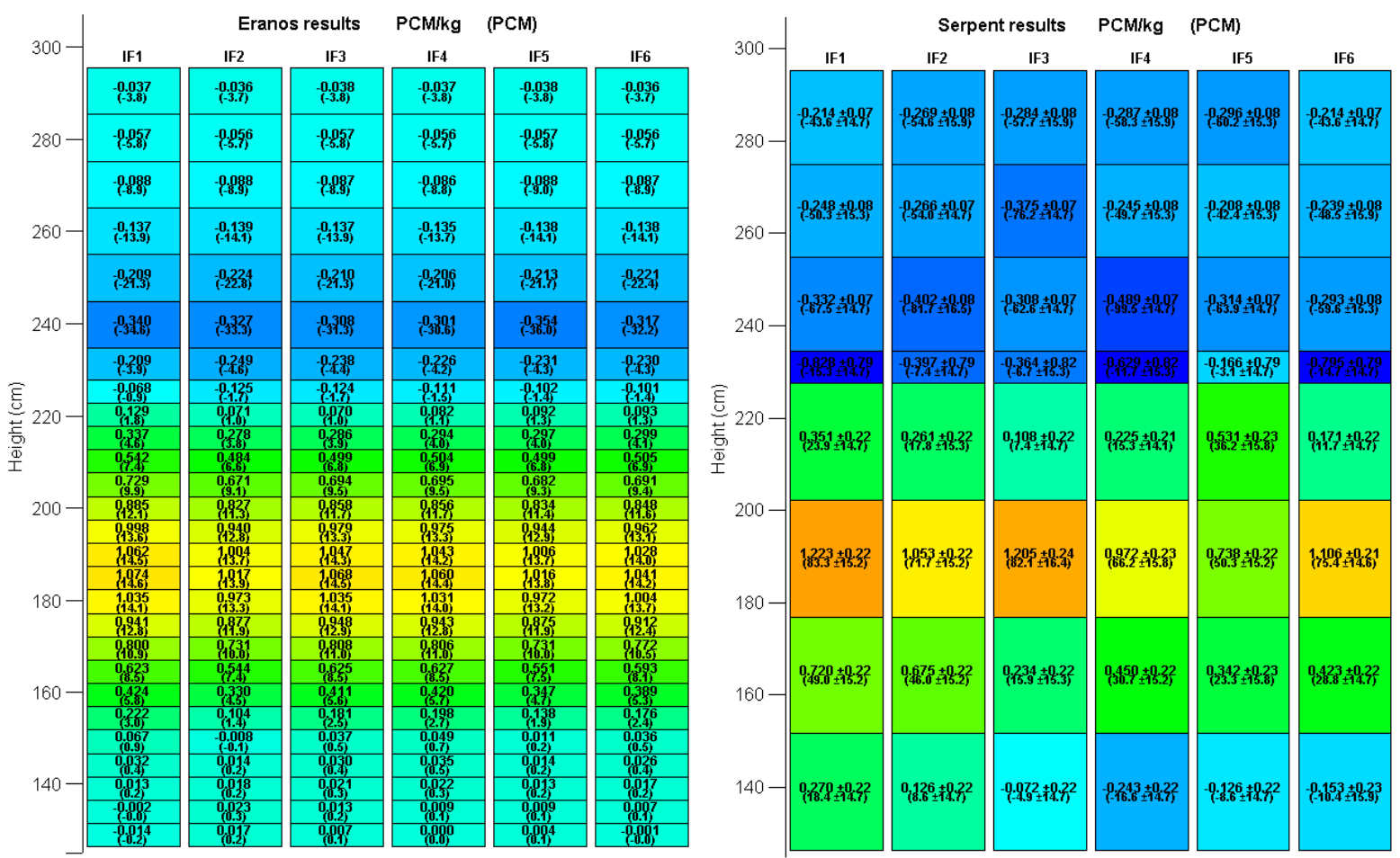

Figure 7. Void reactivity mapping for the six-batch core layout using the full core ERANOS model (left) and the radially infinite Serpent model (right).

\section{ACKNOWLEDGMENTS}

The research leading to these results has received funding from the Euratom research and training program 2014-2018 under grant agreement No 754501.

\section{REFERENCES}

1. Krepel J, Losa E. Closed U-Pu and Th-U cycle in sixteen selected reactors: Comparison of major equilibrium features. Annals of Nuclear Energy. 2019; vol: 128; pp: 341-57.

2. Fiorini GL, Vasile A. European Commission - 7th framework programme: The collaborative project on European sodium fast reactor (CP ESFR). Nuclear Engineering and Design. 2011; vol: 241; pp: 3461-9.

3. Varaine F, Marsault P, Chenaud MS, Bernardin B, Conti A, Sciora P, et al. Pre-conceptual design study of ASTRID core. Proceedings of ICAPP 2012, Chiacago, USA; vol: 1; pp: 572-9.

4. Mikityuk K., et al. "ESFR - SMART: new Horizon - 2020 project on SFR safety." In FR17 International Conference. Yekaterinburg, Russia (2017).

5. Kim YI, Hill R, Grimm K, Rimpault G, Newton T, Li ZH, et al. BN-600 full MOX core benchmark analysis. Proceedings of PHYSOR 2004; pp: 249-61.

6. Krepel J, Pelloni S, Bortot S, Panadero A, Mikityuk K. Mapping of Sodium Void Worth and Doppler Effect for Sodium-cooled Fast Reactor. Proceedings of ICAPP 2015; Nice, France.

7. Tomic G, Krepel J. ESFR-SMART core burnup calculation on radially infinite lattice with Monte-Carlo code. Proceedings of ICAPP 2019, Juan-les-pins, France.

8. Rineiski A, Meriot C, Marchetti M, Krepel J, Iket KIT, Nord C, et al. Core Safety Measures in ESFRSMART. PHYSOR 2018, Cancun, Mexico. pp: 3401-12.

9. Leppanen J. Serpent - a Continuous-energy Monte Carlo Reactor Physics Burnup Calculation Code; VTT Technical Research Centre of Finland. (June 18, 2015).

10. Sisl V, Frybort J, Krepel J, Division S. Mixed LEU - Th Initial Core and Running - In Phase for the HTR PM Reactor. Proceedings of HTR 2018; Warsaw, Poland.

11. Krepel J, Pelloni S, Mikityuk K, Coddington P. EQL3D: ERANOS based equilibrium fuel cycle procedure for fast reactors. Annals of Nuclear Energy. 2009; vol: 36; pp: 550-61.

12. Davies U., as al. Evaluation of the ESFR end of cycle state and detailed analysis of spatial distributions of reactivity coefficients. PHYSOR2020, Cambridge, United Kingdom, March 29th-April 2nd, 2020. 